\title{
Study on the Corrosion Resistancy of Spherical Amalgam with Reference to the Human Saliva
}

\author{
by
}

Kazuo NAGAI* and Masayoshi OHASHI*

Research Collaborators

S. UEHARA, K. MIHARA, K. NEMOTO, T. SHIMIZU,

T. SHIMOJO, F. KORENAGA, E. MASUI and N. GOTO

1. Introduction

2. Method and materials

\subsection{Materials}

2.2 Preparation of test specimens

2.3 Preparatory procedures for experiment

2.3.1 Artificial saliva

2.3.2 Salivae of adults and children

2.3.3 Experimental method

3. Experimental findings

3.1 Corrosive tendency by artificial saliva

3.1.1 Effect on conventional amalgam

3.1.2 Effect on spherical amalgam

3.1.3 Comparison of conventional and spherical amalgams

3.1.4 Summary of corrosive findings

3.2 Corrosive tendency by adult saliva

3.2.1 Effect on conventional amalgam

3.2.2 Effect on spherical amalgam

3.2.3 Comparison of conventional and spherical amalgams

3.3 Corrosive tendency by child saliva

3.3.1 Effect on conventional amalgam

3.3.2 Effect of spherical amalgam

3.3.3 Comparison of conventional and spherical amalgams

4. Discussion

4.1 Differences owing to different kinds of saliva

4.2 Differences in corrosive tendencies of conventional and spherical amalgams

4.3 Effect of different condensation pressures on amalgam corrosion

5. Conclusions

*永井一夫, 大橋 正敬：Dept. of Dental Materials, Nihon Univ. School of Dentistry, Tokyo. 


\section{Introduction}

In 1961, Federal Mogul Bower Bearings, Inc., Detroit, Michigan, U. S. A., was successful in manufacturing spherical particles from an amalgam alloy and was granted the US Patent No. 2,968,062 [1]. It is no undue exaggeration to say that this meant a revolution in the manufacture of amalgam alloys. One year later (1962), DEMAREE and TAYLOR [2] published a result of their study on the particles and various physical properties of this spherical amalgam alloy and following in their wake, KORAN and ASGAR [3] and EDEN and WATERSTRAT [4] respectively gave their research findings of the spherical amalgam alloy later in the same year.

In 1966 NAGAI, OHASHI and MiYAZU [5] published a comprehensive study on the general aspects of spherical amalgam as compared with conventional one and in 1967 NAGAI and OHASHI with collaborators $[6,7,8]$ published further findings on spherical amalgam, these research results paving the way for our better understanding of this important dental restorative material.

In the present study, the authors were chiefly concerned with an application of spherical amalgam to the pedodontic field and directed their particular attention to the comparison of corrosive tendency of spherical amalgam in human saliva, particularly infant saliva with that of artificial saliva.

Hitherto, a principal method for the study of corrosion of dental metals and alloys has been one which examined quantitative changes by immersing them in some kind of corrosive agent. NAGAI and YAMAMOTO[9] recognized advantages of an electrochemical method and further, NAGAI introduced the use of polarography for the purpose of corrosive determination of dental alloys. TSURUMAKI [10], under the guidance of NAGAI, investigated the corrosive tendency of various dental alloys by means of different immersion agents, and HABU [11] of the same Department of Dental Materials, Nihon University School of Dentistry, reported interesting findings on the influence of certain variables of manipulation on amalgam corrosion.

With the use of polarography, whose effectiveness as a tool to determine the corrosion of amalgam quickly and accurately was already demonstrated [11], it was the intention of the authors to evaluate the clinical value of spherical amalgam as compared with conventional amalgam in respect of its better anti-corrosion property and to demonstrate it would be quite suitable to be applied in pedodontics. Corrosive agents used were three kinds of saliva: artificial, adult and infant.

\section{Method and Materials}

\subsection{Materials}

As spherical amalgam, "Spherical amalgam alloy" (batch No. 28), product of Shofu Dental Mfg. Co., was used and for control "Micro non-zinc alloy" (batch No. 42 ) of the same manufacturer was used. These two are to be hereafter referred to as spherical and conventional amalgams respectively. Mercury used was one which is ordinarily used for chemical agent.

\subsection{Preparation of Test Specimens}

Alloy-mercury ratio for conventional amalgam was $1.0: 1.0$ that is accepted as standard by the dental profession, while 1.0:0.85 ratio was adopted for spherical amalgam which has been established as optimal by various tests by NAGAI, OHASHI and 
thier associates. Test specimens prepared in these ratios were subjected to the adult and infant salivae and, as for artificial saliva, a range from $1.0: 0.6$ to $1.0: 1.0$ for the spherical alloy and $1.0: 1.0$ and $1.0: 1.4$ for the conventional alloy were comparatively examined.

Quantitative measurement was effected by the use of Ishida automatic scales with a precision of $1 / 100 \mathrm{mmg}$ and Luna IIB amalgamator was used for trituration (Fig. 1). Alloy powder and mercury which were carefully measured out were placed in a capsule and triturated for 15 seconds. The mixture was mounted on NON condensing apparatus (Fig. 2) without any mercury squeeze; with this apparatus it is possible to obtain varying condensation pressures by merely changing loads [5].

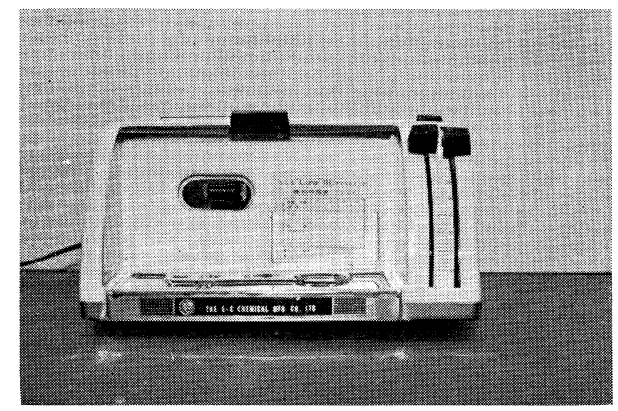

Fig. 1. G-C Luna amalgamator

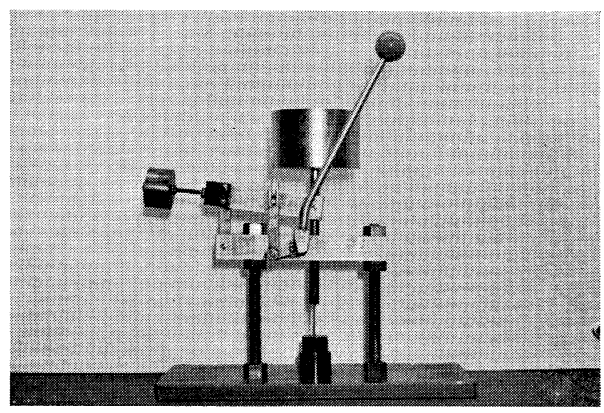

Fig. 2. NON condensing apparatus

After trituration, the amalgam mix was divided into 4 equal parts. One part was put in a metal mold $4.0 \mathrm{~mm}$ in inside diameter and with a plugger $2.5 \mathrm{~mm}$ in diameter, it was so arranged that while the mold rotated 3 times the plugger gave 25 impacts of even distribution. The remaining 3 parts were subjected to the same procedure each, thus 100 impacts being given to the mix. In the last stage, final condensation pressure corresponding to that of $2.5 \mathrm{~mm}$ in diameter was added for 60 seconds. More than 3 minutes and 30 seconds should not be spent from the initiation of trituration to addition of final pressure. After these procedures, the specimen was cut to the length of $8 \mathrm{~mm}$ and was ejected from the mold.

At the addition of final condensation pressure, that part of mercury which was squeezed out of the mix was collected and measured. Although this mercury was by no means pure, it was regarded as representing the residual mercury for the sake of convenience. Throughout the preparation and subsequent storage of test specimens, they were performed at room temperature of $23^{\circ} \pm 2^{\circ} \mathrm{C}$.

2.3 Preparatory Procedures for Experiment.

\subsubsection{Artificial Saliva.}

As the artificial saliva ad modum GREENWOOD [12] is usually employed by our Department for the polarographic determination of corrosion on the part of amalgam alloys, the same was used for the purpose of our basic study here.

\subsubsection{Salivae of Adults and Children.}

In consideration of clinical environments in vivo, we decided on the adoption of mixed saliva at the time of normal rest. For our purposes, both the incoming adult and child patients at the University infirmary were subjected to the collection of their 
salivae prior to the start of treatment, usually between $10: 00$ and $11: 00$ in the morning. Immediately after the collection, the salivae were stored in a cold place and $\mathrm{pH}$ levels were read just before the tests commenced.

The $\mathrm{pH}$ level of child saliva was 6.4 to 7.6 and that of adult was 6.2 to 7.8 with the mean of 7.0 in both cases.

\subsubsection{Experimental Method.}

The surface to come in contact with a corrosive agent was carefully polished in conditions where possible generation of heat was avoided, final polish being given by the use of No. 1000 water-proof sand paper. After 24 hours, a pair of test specimens was placed in an electrolytic vat so that only the contact surfaces were exposed (Fig. 3). The interelectrode distance was $20 \mathrm{~mm}$ and both the electrodes were fully immersed in $2 \mathrm{cc}$ of the corrosive agent and measurements were undertaken at the lapse of 30 seconds. A partial modification of the YANAGIMOTO automatic-recording polarograph P. B. 105 was used (Fig. 4). This apparatus was equipped with an electrical sensitivity

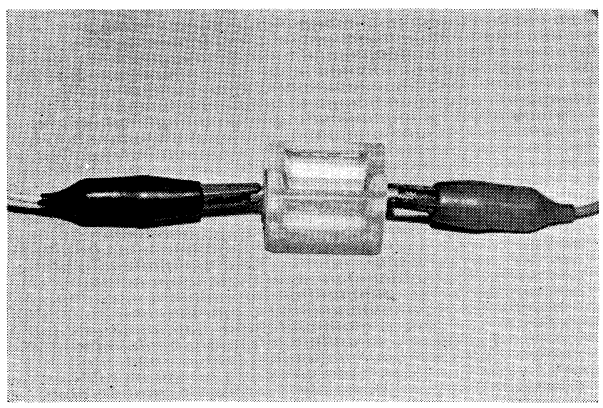

Fig. 3. Electrolytic bath

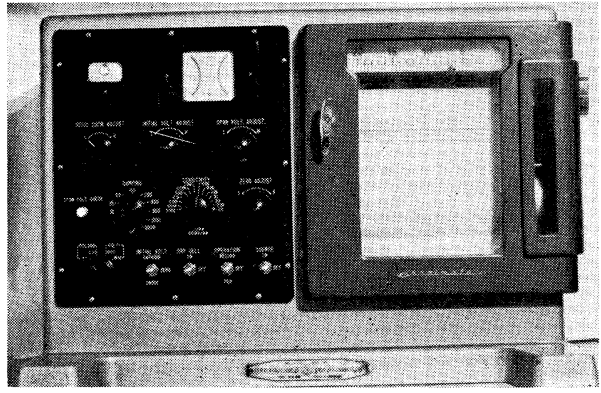

Fig. 4. Polarograph

of $0.05 \mu \mathrm{A} / \mathrm{mm}$ and capable of moving the recording sheet $3 \mathrm{~cm}$. per minute. Current values at a given electric voltage were measured and the lower were the current values, the corrosive resistancy of a specimen was interpreted as correspondingly good. The results to be given below were based on the arithmetic mean of 5 to 10 measurements.

\section{Experimental Findings}

\subsection{Corrosive Tendency by Artificial Saliva.}

\subsubsection{Effect on Conventional Amalgam.}

Fig. 5 to Fig. 7 give the voltage-current readings on conventional amalgam, triturated in a ratio of $1.0: 1.0$, which was condensed under $10 \mathrm{Kg} / \mathrm{cm}^{2}, 30 \mathrm{Kg} / \mathrm{cm}^{2}$ and $60 \mathrm{Kg} / \mathrm{cm}^{2}$ respectively. Fig. 8 to Fig. 10 are concerned with the same conventional amalgam prepared in 1.0:1.4 ratio. Table 1 indicates the measurements of this amalgam in 1.0:1.0 and Table 2 those in 1.0:1.4 packed under each of the above condensation pressures. Table 3 compares the readings at $0.2 \mathrm{~V}$ for convenience.

In common with all the cases, it is definitely established that a smaller mercury ratio (1.0) has less corrosive tendency than a larger one (1.4) and, inversely, the smaller is a condensation pressure, the larger resultant corrosive tendency will result. It is 
also known, in this connection, that the amount of residual mercury increase according to a reduction in condensation pressure and its increase is in parallel to a ratio of

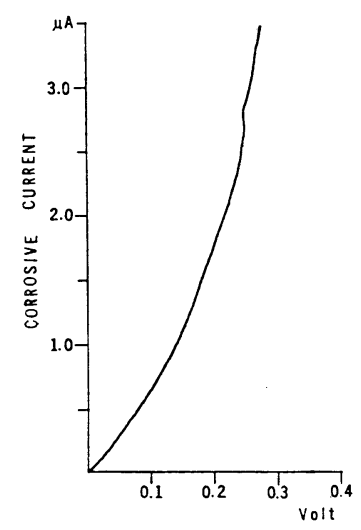

Fig. 5. Conventional $1.0: 1.0 \quad 10 \mathrm{~kg} / \mathrm{cm}^{2}$ Artificial saliva

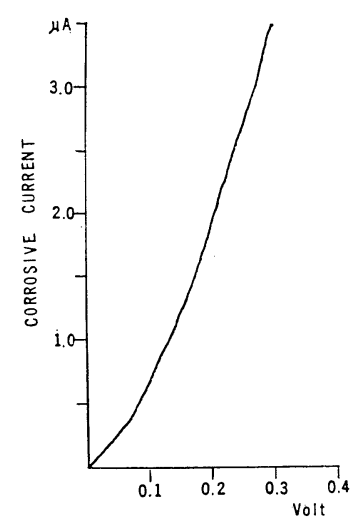

Fig. 8. Conventional $1.0: 1.410 \mathrm{~kg} / \mathrm{cm}^{2}$ Artificial saliva

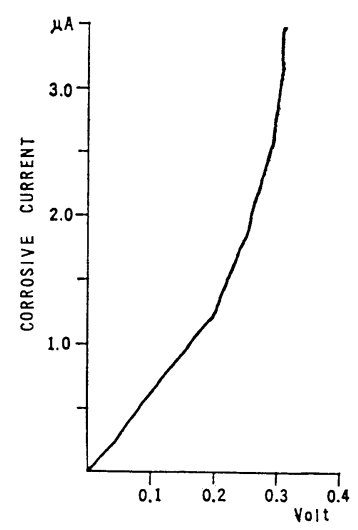

Fig. 6. Conventional $1 \cdot 0: 1.0 \quad 30 \mathrm{~kg} / \mathrm{cm}^{2}$ Artificial saliva

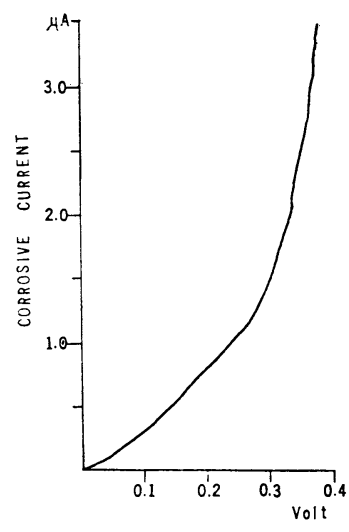

Fig. 9. Conventional $1.0: 1.4 \quad 30 \mathrm{~kg} / \mathrm{cm}^{2}$ Artificial saliva

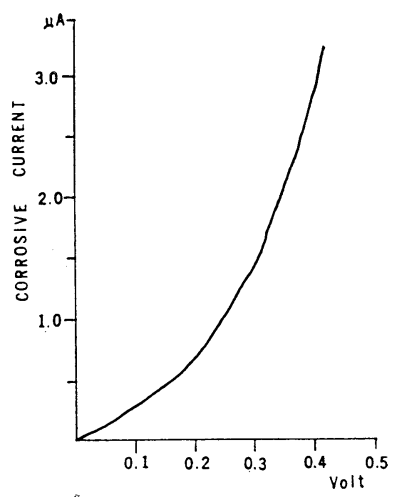

Fig. 7. Conventional $1.0: 1.0 \quad 60 \mathrm{~kg} / \mathrm{cm}^{2}$ Artificial saliva

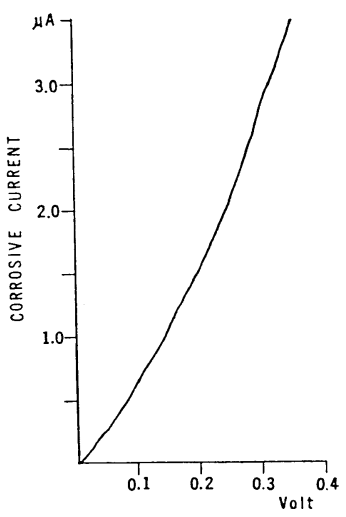

Fig. 10. Conventional $1.0: 1.460 \mathrm{~kg} / \mathrm{cm}^{2}$ Artificial saliva

TABLE 1

Corrosion Current of Conventional Alloy

A-M ratio: $1.0: 1.0$

Artificial saliva

\begin{tabular}{|c|c|c|c|c|}
\hline \multirow{2}{*}{$\begin{array}{l}\text { Condensation } \\
\text { pressure }\end{array}$} & \multicolumn{3}{|c|}{ Voltage } & \multirow{2}{*}{ Residual mercury } \\
\hline & $0.1 \mathrm{v}$ & $0.2 \mathrm{v}$ & $0.3 \mathrm{v}$ & \\
\hline $10 \mathrm{~kg} / \mathrm{cm}^{2}$ & $0.67 \mu \mathrm{A}$ & $1.79 \mu \mathrm{A}$ & $4.58 \mu \mathrm{A}$ & $50.0 \%$ \\
\hline 30 & 0.58 & 1.40 & 3.21 & 48.1 \\
\hline 60 & 0.43 & 0.95 & 2.00 & 46.0 \\
\hline
\end{tabular}


TABLE 2

Corrosion Current of Conventional Alloy

A-M ratio : $1.0: 1.4$

Artificial saliva

\begin{tabular}{l|l|l|l|l}
\hline \hline \multirow{2}{*}{$\begin{array}{c}\text { Condensation } \\
\text { pressure }\end{array}$} & \multicolumn{3}{|c|}{ Voltage } & \multirow{2}{*}{ Reaidual mercury } \\
\cline { 2 - 3 } & $0.1 \mathrm{v}$ & $0.2 \mathrm{v}$ & $0.3 \mathrm{v}$ & \\
\hline $10 \mathrm{~kg} / \mathrm{cm}^{2}$ & $0.69 \mu \mathrm{A}$ & $1.90 \mu \mathrm{A}$ & $3.92 \mu \mathrm{A}$ & $56.1 \%$ \\
30 & 0.58 & 1.41 & 3.02 & 54.5 \\
60 & 0.50 & 1.22 & 2.73 & 53.3
\end{tabular}

TABLE 3

Corrosion Current of Conventional Alloy

Summation at 0.2 Volt.

Artificial saliva

\begin{tabular}{l|c|c|c|c}
\hline \hline \multirow{2}{*}{$\begin{array}{c}\text { Condensation } \\
\text { pressure }\end{array}$} & A-M ratio & \multirow{2}{*}{$\begin{array}{c}\text { Residual } \\
\text { mercury }\end{array}$} & A-M ratio & \multirow{2}{*}{ Residual mercury } \\
\cline { 2 - 2 } & $1.0: 1.0$ & & $1.0: 1.4$ & \\
\hline $10 \mathrm{~kg} / \mathrm{cm}^{2}$ & $1.79 \mu \mathrm{A}$ & $50.0 \%$ & $1.90 \mu \mathrm{A}$ & $56.1 \%$ \\
30 & 1.40 & 48.1 & 1.41 & 54.5 \\
60 & 0.95 & 46.0 & 1.22 & 53.3
\end{tabular}

alloy-mercury. Residual mercury of an amalgam alloy triturated in a ratio of $1.0: 1.0$ under a condensation pressure of $10 \mathrm{Kg} / \mathrm{cm}^{2}$ was $50 \%$, which would represent the amount of mercury in the test specimen at the time of trituration as it could not be squeezed out under the addition of final load.

\subsubsection{Effcet on Spherical Amalgam.}

As for spherical amalgam specimens, they were prepared in a alloy-mercury ratio range from $1.0: 0.6$ to $1.0: 1.0$ under four condensation pressures of $5 \mathrm{Kg} / \mathrm{cm}^{2}$, $10 \mathrm{Kg} / \mathrm{cm}^{2}, 30 \mathrm{Kg} / \mathrm{cm}^{2}$ and $60 \mathrm{Kg} / \mathrm{cm}^{2}$. Figs. 11 to 38 give voltage-current curves on these various test pieces. Their breakdown is 1.0:0.6 (Figs. 11-14), 1.0:0.7 (Figs. 15-18), $1.0: 0.75$ (Figs. 19-22), $1.0: 0.80$ (Figs. 23-26), $1.0: 0.85$ (Figs. 27-30), $1.0: 0.9$ (Figs. 31-34) and 1.0:1.0 (Figs. 35-38). On the other hand, Tables 4 to 10 compare the measurements of spherical amalgam specimens that were triturated in different alloymercury ratios under $5 \mathrm{Kg} / \mathrm{cm}^{2}, 10 \mathrm{Kg} / \mathrm{cm}^{2}, 30 \mathrm{Kg} / \mathrm{cm}^{2}$ and $60 \mathrm{Kg} / \mathrm{cm}^{2}$ condensation pressures. Table 11 gives the voltage-current readings as $0.2 \mathrm{~V}$ as in 3.1 .1 . It is noted from this that within a ratio range from $1.0: 0.7$ to 0.85 there is the least reading in the corrosive pattern of spherical amalgam and, in this connection, what is more interesting is the fact that no appreciable change is apparent within a range of $10 \mathrm{Kg} /$ $\mathrm{cm}^{2}$ to $60 \mathrm{Kg} / \mathrm{cm}^{2}$ condensation pressures. 


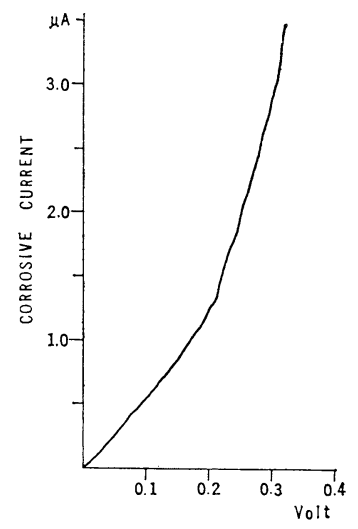

Fig. 11. Spherical $1.0: 0.6 \quad 5 \mathrm{~kg} / \mathrm{cm}^{2}$ Artificial saliva

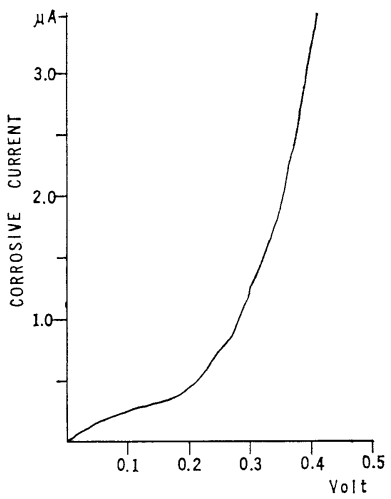

Fig. 14. Spherical $1.0: 0.6 \quad 60 \mathrm{~kg} / \mathrm{cm}^{2}$ Artificial saliva

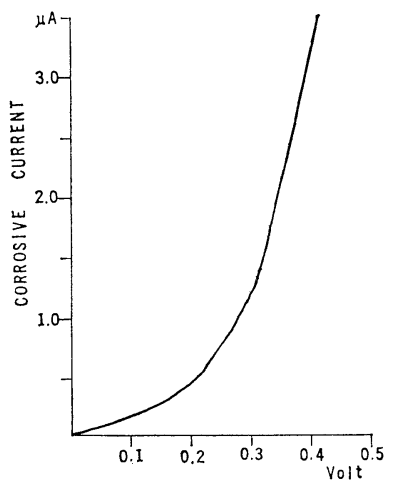

Fig. 17. Spherical $1.0: 0.70 \quad 30 \mathrm{~kg} / \mathrm{cm}^{2}$ Artificial saliva

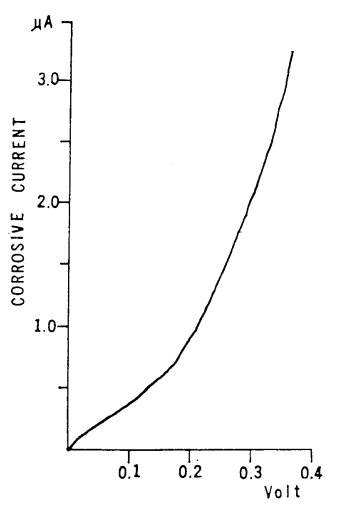

Fig. 12. Spherical $1.0: 0.610 \mathrm{~kg} / \mathrm{cm}^{2}$ Artificial saliva

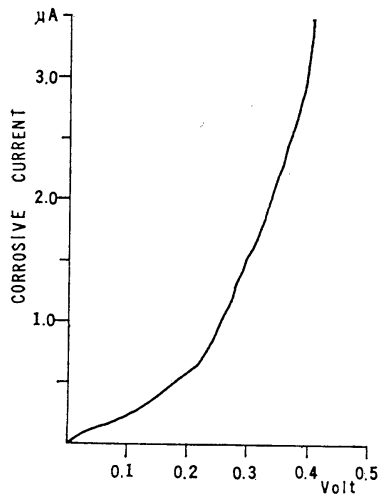

Fig. 15. Spherical $1.0: 0.70 \quad 5 \mathrm{~kg} / \mathrm{cm}^{2}$ Artificial saliva

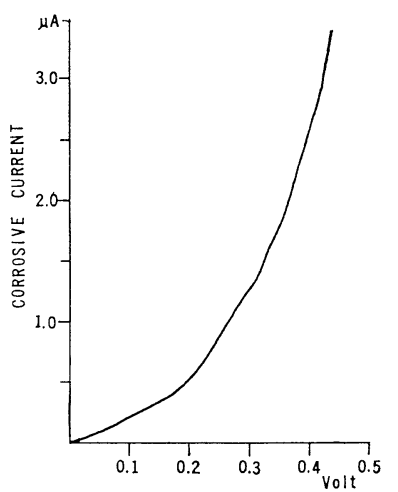

Fig. 18. Spherical $1.0: 0.70 \quad 60 \mathrm{~kg} / \mathrm{cm}^{2}$ Artificial saliva

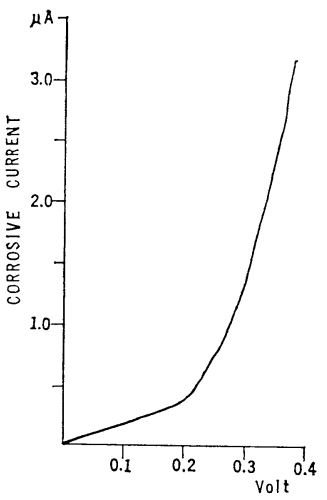

Fig. 13. Spherical $1.0: 0.6 \quad 30 \mathrm{~kg} / \mathrm{cm}^{2}$ Artificial saliva

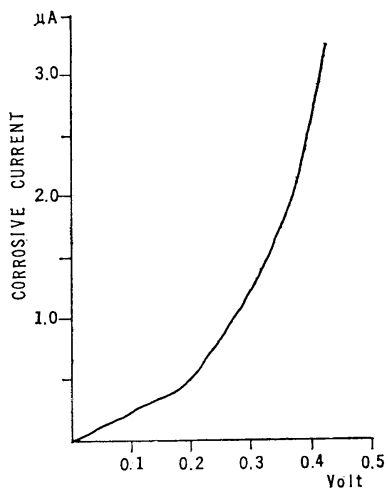

Fig. 16. Spherical $1.0: 0.70 \quad 10 \mathrm{~kg} / \mathrm{cm}^{2}$ Artificial saliva

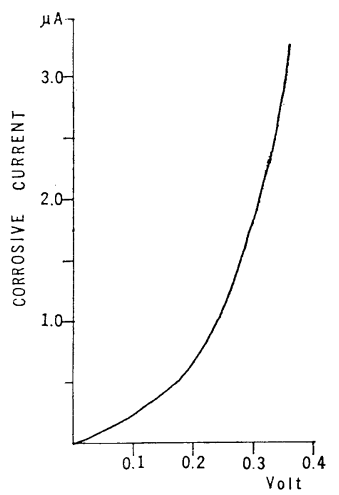

Fig. 19. Spherical $1.0: 0.75 \quad 5 \mathrm{~kg} / \mathrm{cm}^{2}$ Artificial saliva 


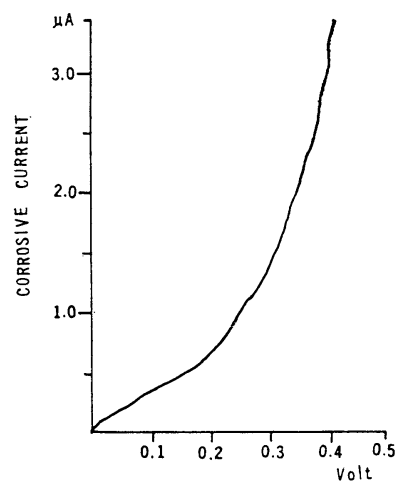

Fig. 20. Spherical $1.0: 0.7510 \mathrm{~kg} / \mathrm{cm}^{2}$ Artificial saliva

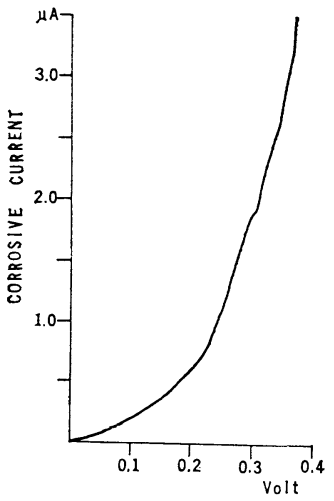

Fig. 23. Spherical $1.0: 0.80 \quad 5 \mathrm{~kg} / \mathrm{cm}^{2}$ Artificial saliva

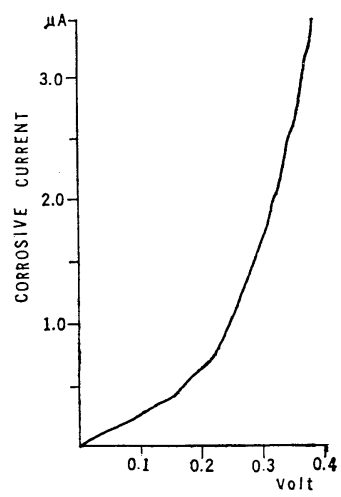

Fig. 26. Spherical $1.0: 0.80 \quad 60 \mathrm{~kg} / \mathrm{cm}^{2}$ Artificial saliva

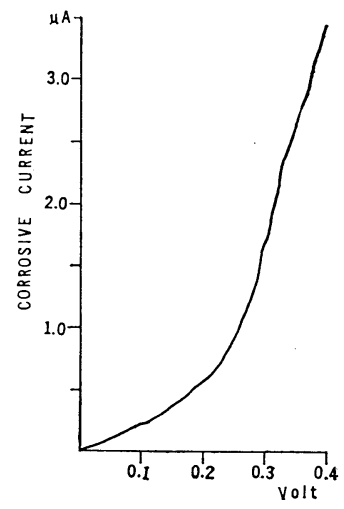

Fig. 21. Spherical $1.0: 0.7530 \mathrm{~kg} / \mathrm{cm}^{2}$ Artificial saliva

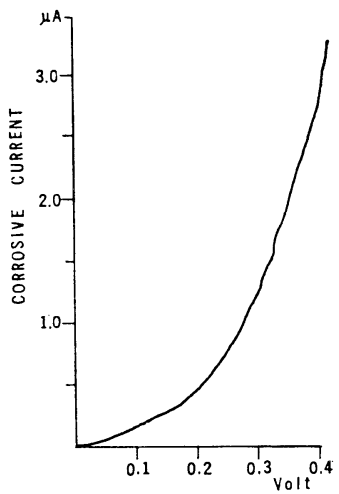

Fig. 24. Spherical $1.0: 0.80 \quad 10 \mathrm{~kg} / \mathrm{cm}^{2}$ Artificial saliva

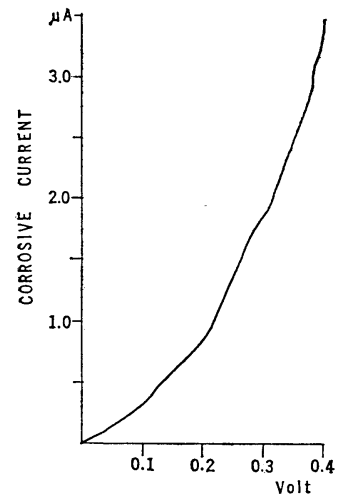

Fig. 27. Spherical $1.0: 0.855 \mathrm{~kg} / \mathrm{cm}^{2}$ Artificial saliva

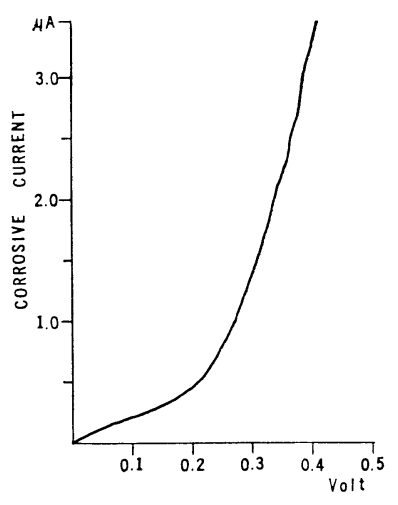

Fig. 22. Spherical $1.0: 0.75 \quad 60 \mathrm{~kg} / \mathrm{cm}^{2}$ Artificial saliva

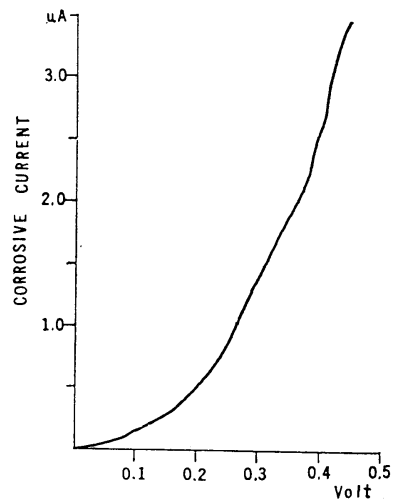

Fig. 25. Spherical $1.0: 0.80 \quad 30 \mathrm{~kg} / \mathrm{cm}^{2}$ Artificial saliva

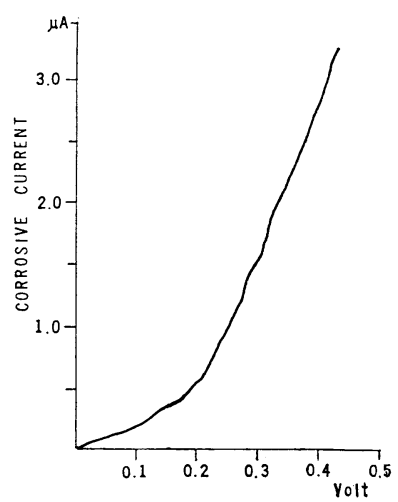

Fig. 28. Spherical $1.0: 0.8510 \mathrm{~kg} / \mathrm{cm}^{2}$ Artificial saliva 


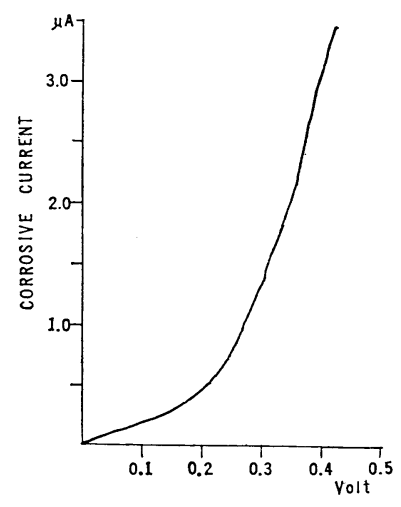

Fig. 29. Spherical $1.0: 0.8530 \mathrm{~kg} / \mathrm{cm}^{2}$ Artificial saliva

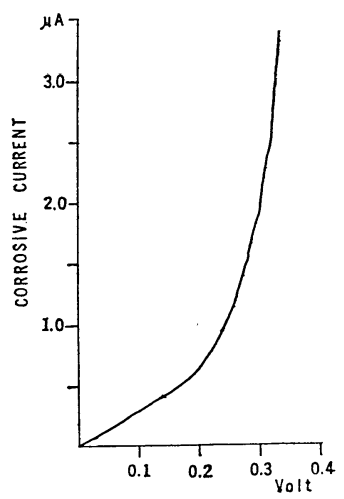

Fig. 32. Spherical $1.0: 0.910 \mathrm{~kg} / \mathrm{cm}^{2}$ Artificial saliva

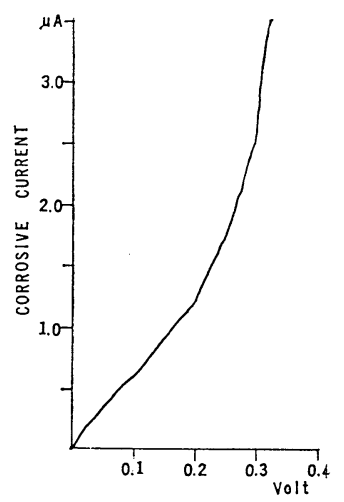

Fig. 35. Spherical $1.0: 1.0 \quad 5 \mathrm{~kg} / \mathrm{cm}^{2}$ Artificial saliva

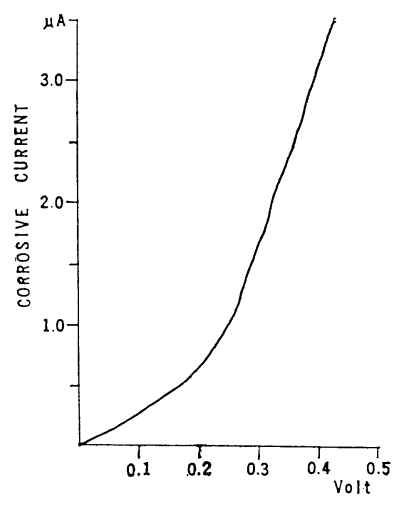

Fig. 30. Spherical $1.0: 0.8560 \mathrm{~kg} / \mathrm{cm}^{2}$ Artificial saliva

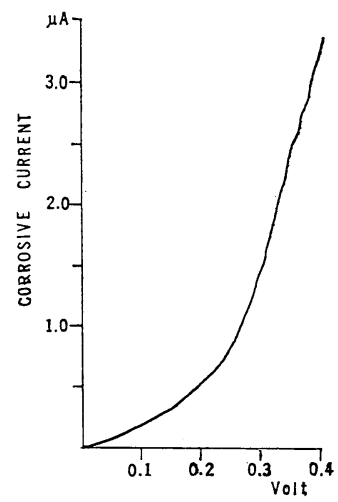

Fig. 33. Spherical $1.0: 0.930 \mathrm{~kg} / \mathrm{cm}^{2}$ Artificial saliva

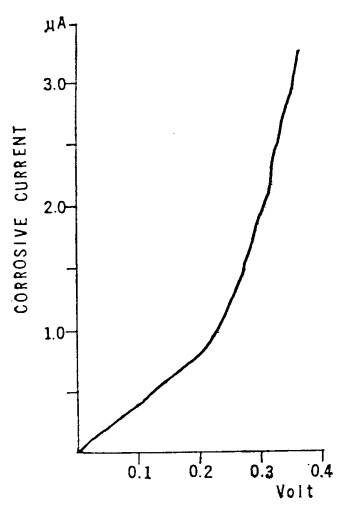

Fig. 31. Spherical $1.0: 0.95 \mathrm{~kg} / \mathrm{cm}^{2}$ Artificial saliva

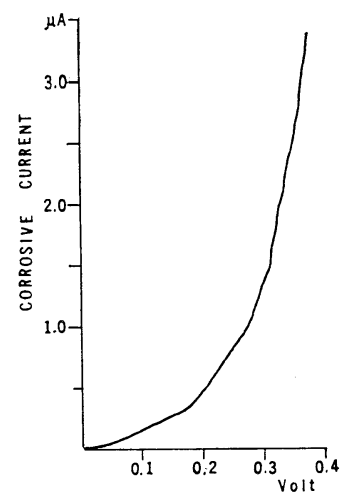

Fig. 34. Spherical $1.0: 0.960 \mathrm{~kg} / \mathrm{cm}^{2}$ Artificial saliva

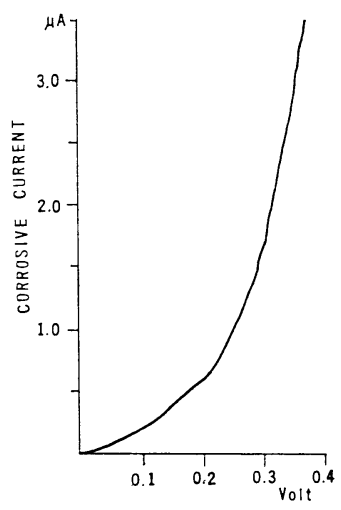

Fig. 36. Spherical $1.0: 1.0 \quad 10 \mathrm{~kg} / \mathrm{cm}^{2}$ Artificial saliva 


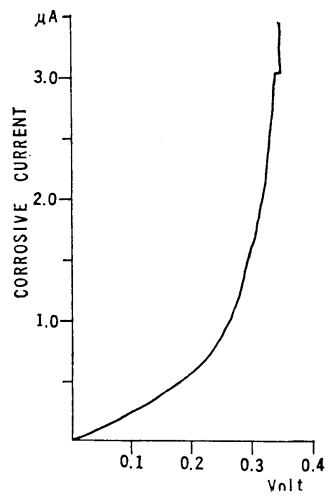

Fig. 37. Spherical $1.0: 1.0 \quad 30 \mathrm{~kg} / \mathrm{cm}^{2}$ Artificial saliva

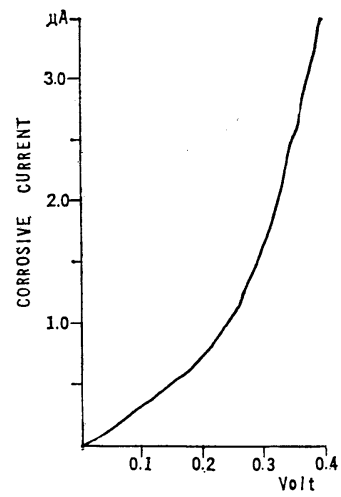

Fig. 38. Spherical $1.0: 1.0 \quad 60 \mathrm{~kg} / \mathrm{cm}^{2}$ Artificial saliva

TABLE 4

Corrosion Current of Spherical Alloy

A-M ratio: $1.0: 0.6$

Artificial saliva

\begin{tabular}{c|l|l|l|c}
\hline \hline \multirow{2}{*}{$\begin{array}{c}\text { Condensation } \\
\text { pressure }\end{array}$} & \multicolumn{3}{|c|}{ Voltage } & Residual mercury \\
\cline { 2 - 3 } & $0.1 \mathrm{v}$ & $0.2 \mathrm{v}$ & $0.3 \mathrm{v}$ & \\
\hline $5 \mathrm{~kg} / \mathrm{cm}^{2}$ & $0.52 \mu \mathrm{A}$ & $1.21 \mu \mathrm{A}$ & $2.81 \mu \mathrm{A}$ & $37.5 \%$ \\
10 & 0.41 & 0.99 & 2.00 & 37.5 \\
30 & 0.32 & 0.78 & 1.42 & 37.5 \\
60 & 0.32 & 0.78 & 1.32 & 37.5
\end{tabular}

TABLE 5

Corrosion Current of Spherical Alloy

A-M ratio: $1.0: 0.7 \quad$ Artificial saliva

\begin{tabular}{c|c|c|c|c}
\hline \hline \multirow{2}{*}{$\begin{array}{c}\text { Condensation } \\
\text { pressure }\end{array}$} & \multicolumn{3}{|c|}{ Voltage } & Residual mercury \\
\cline { 2 - 4 } & $0.1 \mathrm{v}$ & $0.2 \mathrm{v}$ & $0.3 \mathrm{v}$ & \\
\hline $5 \mathrm{~kg} / \mathrm{cm}^{2}$ & $0.32 \mu \mathrm{A}$ & $0.58 \mu \mathrm{A}$ & $1.55 \mu \mathrm{A}$ & $41.2 \%$ \\
10 & 0.31 & 0.52 & 1.55 & 41.2 \\
30 & 0.29 & 0.50 & 1.33 & 40.8 \\
60 & 0.30 & 0.50 & 1.35 & 40.1
\end{tabular}


TABLE 6

Corrosion Current of Spherical Alloy

A-M ratio : $1.0: 0.75$

Artificial saliva

\begin{tabular}{c|l|l|l|l}
\hline \hline \multirow{2}{*}{$\begin{array}{c}\text { Condensation } \\
\text { pressure }\end{array}$} & \multicolumn{3}{|c|}{ Voltage } & \multirow{2}{*}{ Residual mercury } \\
\cline { 2 - 3 } & $0.1 \mathrm{v}$ & $0.2 \mathrm{v}$ & $0.3 \mathrm{v}$ & \\
\hline $5 \mathrm{~kg} / \mathrm{cm}^{2}$ & $0.31 \mu \mathrm{A}$ & $0.57 \mu \mathrm{A}$ & $1.55 \mu \mathrm{A}$ & $43.0 \%$ \\
10 & 0.30 & 0.52 & 1.33 & 43.0 \\
30 & 0.29 & 0.48 & 1.31 & 42.6 \\
60 & 0.29 & 0.49 & 1.34 & 41.7
\end{tabular}

TABLE 7

Corrosion Current of Spherical Alloy

A-M. ratio : $1.0: 0.8$

Artificial saliva

\begin{tabular}{c|l|l|l|l}
\hline \hline \multirow{2}{*}{$\begin{array}{c}\text { Condensation } \\
\text { pressure }\end{array}$} & \multicolumn{3}{|c|}{ Voltage } & \multirow{2}{*}{ Residual mercury } \\
\cline { 2 - 4 } & $0.1 \mathrm{v}$ & $0.2 \mathrm{v}$ & $0.3 \mathrm{v}$ & \\
\hline $5 \mathrm{~kg} / \mathrm{cm}^{2}$ & $0.25 \mu \mathrm{A}$ & $0.51 \mu \mathrm{A}$ & $1.73 \mu \mathrm{A}$ & $44.0 \%$ \\
10 & 0.20 & 0.49 & 1.43 & 44.0 \\
30 & 0.20 & 0.47 & 1.39 & 43.5 \\
60 & 0.21 & 0.49 & 1.41 & 41.6
\end{tabular}

TABLE 8

Corrosion Current of Spherical Alloy

A-M ratio: $1.0: 0.85$

Artificial saliva

\begin{tabular}{c|l|l|l|l}
\hline \hline \multirow{2}{*}{$\begin{array}{c}\text { Condensation } \\
\text { pressure }\end{array}$} & \multicolumn{3}{|c|}{ Voltage } & \multirow{2}{*}{ Residual mercury } \\
\cline { 2 - 4 } & $0.1 \mathrm{v}$ & $0.2 \mathrm{v}$ & $0.3 \mathrm{v}$ & \\
\hline $5 \mathrm{~kg} / \mathrm{cm}^{2}$ & $0.27 \mu \mathrm{A}$ & $0.56 \mu \mathrm{A}$ & $1.91 \mu \mathrm{A}$ & $46.0 \%$ \\
10 & 0.23 & 0.51 & 1.52 & 45.8 \\
30 & 0.21 & 0.48 & 1.41 & 44.7 \\
60 & 0.22 & 0.50 & 1.42 & 43.5
\end{tabular}

TABLE 9

Corrosion Current of Spherical Alloy

A-M ratio: $1.0: 0.9$

Artificial saliva

\begin{tabular}{c|l|l|l|l}
\hline \hline \multirow{2}{*}{$\begin{array}{c}\text { Condensation } \\
\text { pressure }\end{array}$} & \multicolumn{3}{|c|}{ Voltage } & \multirow{2}{*}{ Residual mercury } \\
\cline { 2 - 3 } & $0.1 \mathrm{v}$ & $0.2 \mathrm{v}$ & $0.3 \mathrm{v}$ & \\
\hline $5 \mathrm{~kg} / \mathrm{cm}^{2}$ & $0.41 \mu \mathrm{A}$ & $0.70 \mu \mathrm{A}$ & $1.98 \mu \mathrm{A}$ & $47.4 \%$ \\
10 & 0.40 & 0.69 & 1.79 & 45.8 \\
30 & 0.32 & 0.60 & 1.73 & 44.0 \\
60 & 0.33 & 0.61 & 1.74 & 43.1
\end{tabular}


TABLE 10

Corrosion Current of Spherical Alloy

A-M ratio: $1.0: 1.0$

Artificial saliva

\begin{tabular}{c|l|l|l|c}
\hline \hline \multirow{2}{*}{$\begin{array}{c}\text { Condensation } \\
\text { pressure }\end{array}$} & \multicolumn{3}{|c|}{ Voltage } & \multirow{2}{*}{ Residual mercury } \\
\cline { 2 - 3 } & $0.1 \mathrm{v}$ & $0.2 \mathrm{v}$ & $0.3 \mathrm{v}$ & \\
\cline { 2 - 3 } $5 \mathrm{~kg} / \mathrm{cm}^{2}$ & $0.39 \mu \mathrm{A}$ & $0.71 \mu \mathrm{A}$ & $1.99 \mu \mathrm{A}$ & $49.7 \%$ \\
10 & 0.36 & 0.69 & 1.79 & 49.4 \\
30 & 0.35 & 0.61 & 1.75 & 47.2 \\
60 & 0.35 & 0.61 & 1.76 & 45.6
\end{tabular}

TABLE 11

Corrosion Current of Spherical Alloy

Summation at 0.2 Volt.

\begin{tabular}{c|r|r|r|r|r|r|r}
\hline \hline \multirow{2}{*}{$\begin{array}{c}\text { Condensation } \\
\text { pressure }\end{array}$} & \multicolumn{7}{|c|}{ Mercury ratio against 1 part of alloy } \\
\cline { 2 - 9 } & 0.6 & 0.7 & 0.75 & 0.8 & 0.85 & 0.9 & 1.0 \\
\hline \multirow{2}{*}{5} & 1.21 & 0.58 & 0.57 & 0.51 & 0.56 & 0.70 & 0.71 \\
& $(37.5)$ & $(41.2)$ & $(43.0)$ & $(44.0)$ & $(46.0)$ & $(47.4)$ & $(49.7)$ \\
10 & 0.99 & 0.52 & 0.52 & 0.49 & 0.51 & 0.69 & 0.69 \\
& $(37.5)$ & $(41.2)$ & $(43.0)$ & $(44.0)$ & $(45.8)$ & $(45.8)$ & $(49.2)$ \\
30 & 0.78 & 0.50 & 0.48 & 0.47 & 0.48 & 0.60 & 0.61 \\
& $(37.5)$ & $(40.8)$ & $(42.6)$ & $(43.5)$ & $(44.7)$ & $(44.0)$ & $(47.2)$ \\
60 & 0.78 & 0.50 & 0.49 & 0.49 & 0.50 & 0.61 & 0.61 \\
& $(37.5)$ & $(40.1)$ & $(41.7)$ & $(41.6)$ & $(43.5)$ & $(43.1)$ & $(45.6)$ \\
\hline
\end{tabular}

Unit by $\mathrm{kg} / \mathrm{cm}^{2}$

Upper numbers indicate corrosion current by $\mu \mathrm{A}$.

Lower numbers indicate residual mercury in $\%$.

\subsubsection{Comparison of Conventional and Spherical Amalgams.}

Fig. 39 compares the corrosive tendencies of conventional and spherical amalgam in terms of different packing pressures. Fig. 40 is similarly concerned with a comparison of different alloy-mercury ratios. Examination of these two figures reveals that spherical amalgam has a distinct advantage over conventional in higher corrosiveresistancy. As compared with spherical, conventional amalgam has a generally large corrosive tendency, which rapidly increases proportionally to a reduction in condensation pressure. On the other hand, spherical amalgam has a generally low corrosive tendency and there is seen no appreciable change in the corrosion within a range of $1.0: 0.70$ to 0.85 alloy-mercury ratios. Clinical advantage of spherical amalgam can be seen in the fact that, while the corrosive tendency of spherical amalgam (prepared in $1.0: 0.85$ under $5 \mathrm{Kg} / \mathrm{cm}^{2}$ condensation pressure) registers $0.56 \mu \mathrm{A}$ at $0.2 \mathrm{~V}$, that of conventional amalgam (prepared in $1.0: 1.0$ under $60 \mathrm{Kg} / \mathrm{cm}^{2}$ pressure) is $0.95 \mu \mathrm{A}$ at the same valtage level.

\subsubsection{Summary of Corrosive Findings.}

This part of our studies on the corrosive tendencies of spherical and conventional 


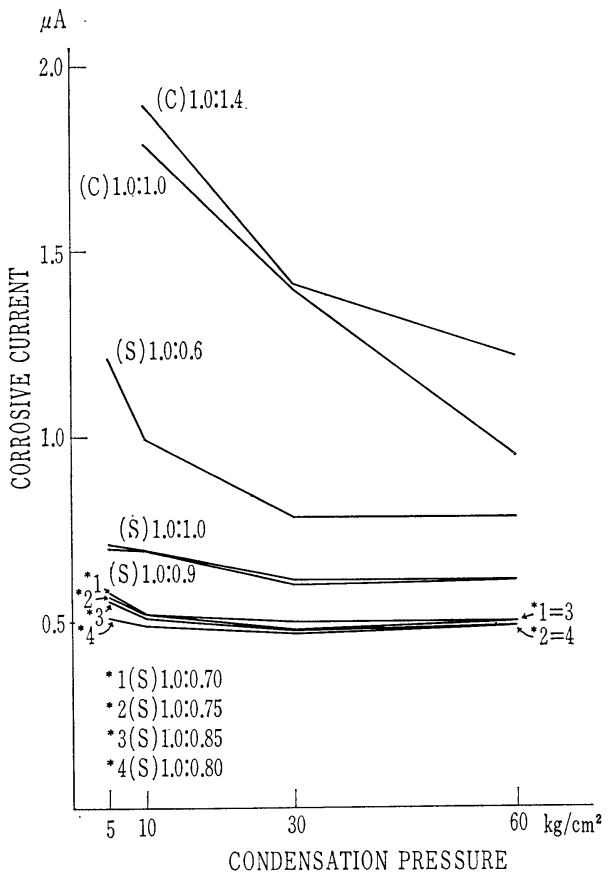

Fig. 39. Comparison of corrosive current of spherical and conventional alloy in artificial saliva, accrording to the change of condensation pressure

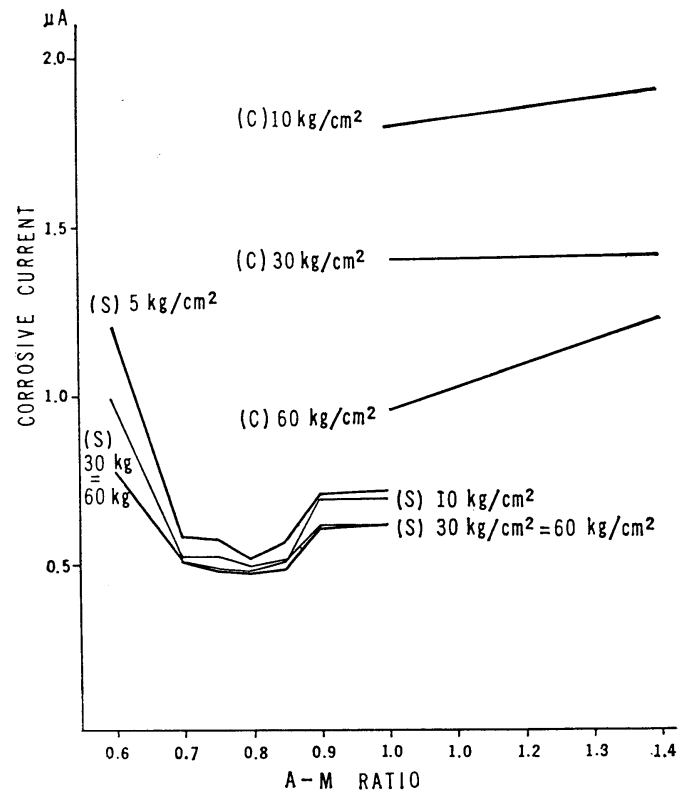

Fig. 40. Comparison of corrosion current of spherical and conventional alloy in artificial saliva, according to the change of $\mathrm{A}-\mathrm{M}$ ratio

amalgam gave the conclusions as follows.

1) Spherical amalgam possesses a higher corrosive-resistancy than conventional.

2) With spherical amalgam, there is no appreciable change in its corrosive pattern within a range of $1.0: 0.7$ to 0.85 alloy-mercury ratios.

3) With spherical amalgam, a range of 10 to $60 \mathrm{Kg} / \mathrm{cm}^{2}$ condensation pressures has no effect in changing its corrosive pattern.

4) Conventional amalgam is greatly influenced by changes in condensation pressure and alloy-mercury ratio as regards its corrosive pattern.

\subsection{Corrosive Tendency by Adult Saliva.}

\subsubsection{Effect on Conventional Amalgam.}

Figs. 41 and 42 respectively give the voltage-current curves of conventional amalgam prepared in $1.0: 1.0$ and packed under the pressure of $10 \mathrm{Kg} / \mathrm{cm}^{2}$ and $60 \mathrm{Kg} / \mathrm{cm}^{2}$. Further, Table 12 compares the mean current readings under these two packing pressures. Here those specimens packed under a low pressure gave a higher corrosive tendency.

\subsubsection{Effect on Spherical Amalgam.}

Figs. 43 and 44 respectively give the voltage-current curves of spherical amalgam packing under the same pressures but prepared in 1.0:0.85 ratio. Table 13 compares the mean current readings. The corrosive tendency here is somewhat low generally as 
against the artificial saliva and, as is the case with artificial, human saliva has also no appreciable effect on changes in the corrosive tendency within a range of 10 to $60 \mathrm{Kg} / \mathrm{cm}^{2}$ condensation pressures.

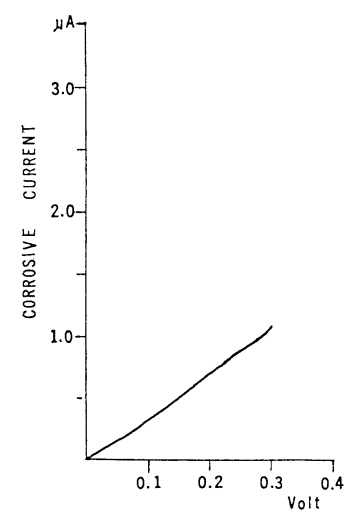

Fig. 41. Conventional $1.0: 1.0 \quad 10 \mathrm{~kg} / \mathrm{cm}^{2}$ Adult saliva

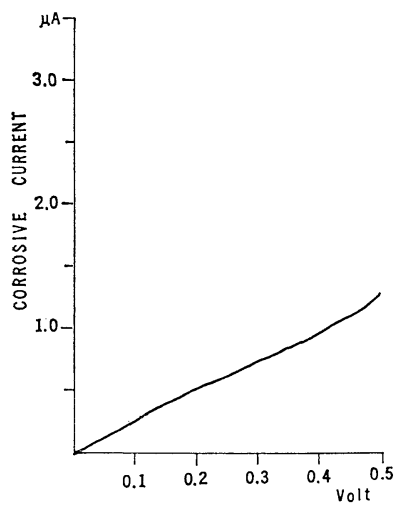

Fig. 43. Spherical $1.0: 0.85 \quad 10 \mathrm{~kg} / \mathrm{cm}^{2}$ Adult saliva

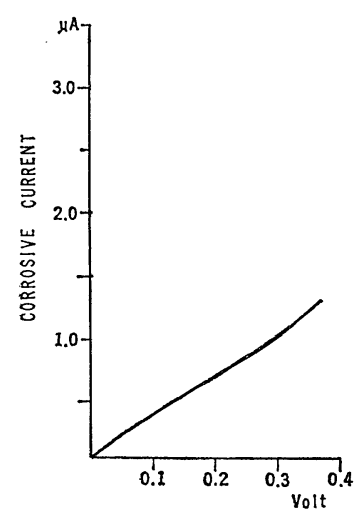

Fig. 42. Conventional $1.0: 1.0 \quad 60 \mathrm{~kg} / \mathrm{cm}^{2}$ Adult saliva

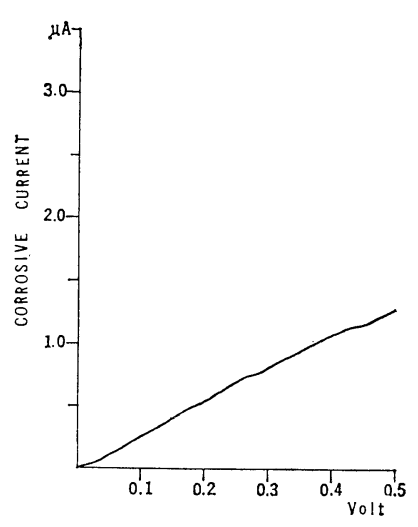

Fig. 44. Spherical $1.0: 0.8560 \mathrm{~kg} / \mathrm{cm}^{2}$ Adult saliva

TABLE 12

Corrosion Current of Conventional Alloy A-M ratio : $1.0: 1.0$ Adult saliva

\begin{tabular}{|c|c|c|c|c|}
\hline \multirow{2}{*}{$\begin{array}{l}\text { Condensation } \\
\text { pressure }\end{array}$} & \multicolumn{3}{|c|}{ Voltage } & \multirow{2}{*}{ Residual mercury } \\
\hline & $0.1 \mathrm{v}$ & $0.2 \mathrm{v}$ & $0.3 \mathrm{v}$ & \\
\hline $\begin{array}{l}10 \mathrm{~kg} / \mathrm{cm}^{2} \\
60\end{array}$ & $\begin{array}{l}0.40 \mu \mathrm{A} \\
0.38\end{array}$ & $\begin{array}{l}0.79 \mu \mathrm{A} \\
0.68\end{array}$ & $\begin{array}{l}1.26 \mu \mathrm{A} \\
0.96\end{array}$ & $\begin{array}{l}50.0 \% \\
46.0\end{array}$ \\
\hline
\end{tabular}


TABLE 13

Corrosion Current of Spherical Alloy

A-M ratio : $1.0: 0.85$

Adult saliva

\begin{tabular}{c|c|c|c|c}
\hline \hline \multirow{2}{*}{$\begin{array}{c}\text { Condensation } \\
\text { pressure }\end{array}$} & \multicolumn{2}{|c|}{ Voltage } & Residual mercury \\
\cline { 2 - 3 } & $0.1 \mathrm{v}$ & $0.2 \mathrm{v}$ & $0.3 \mathrm{v}$ & \\
\hline $10 \mathrm{~kg} / \mathrm{cm}^{2}$ & $0.31 \mu \mathrm{A}$ & $\begin{array}{l}0.54 \mu \mathrm{A} \\
0.52\end{array}$ & $\begin{array}{l}0.79 \mu \mathrm{A} \\
0.80\end{array}$ & $\begin{array}{l}45.8 \% \\
43.5\end{array}$ \\
\hline 60 & 0.33 & &
\end{tabular}

\subsubsection{Comparison of Conventional and Spherical Amalgams.}

With the adult saliva as a corrosive agent, Table 14 gives a comparison of the corrosive tendencies on the part of conventional and spherical amalgams at $0.2 \mathrm{~V}$. As is learned from the comparison, while the corrosive tendency of spherical remains more or less unchanged within a range of 10 to $60 \mathrm{Kg} / \mathrm{cm}^{2}$ condensation pressure, that of conventional amalgam is greatly influenced by whether a condensation pressure is large or small, the corrosive tendency rapidly growing proportionally to the reduction in condensation pressure. Spherical registers only $0.54 \mu \mathrm{A}$ when packed under a pressure as low as $10 \mathrm{Kg} / \mathrm{cm}^{2}$ but conventional amalgam gives $0.68 \mu \mathrm{A}$ even when it is packed under six times large, $60 \mathrm{Kg} / \mathrm{cm}^{2}$.

\subsection{Corrosive Tendency by Child Saliva.}

\subsubsection{Effect on Conventional Amalgam.}

The same alloy-mercury ratio of 1.0:1.0 was adopted as in the case of adult saliva and the measurements were made under two condensation pressures of $10 \mathrm{Kg} / \mathrm{cm}^{2}$ $60 \mathrm{Kg} / \mathrm{cm}^{2}$ (Figs. 45, 46). Table 15 gives a comparison of the two, where a lower and condensation pressure reveals a larger corrosive tendency.

\section{TABLE 14}

Comparison of Corrosion Current of Spherical and Conventional Alloy at 0.2 volt.

Adult saliva

\begin{tabular}{|c|c|c|}
\hline Condensation pressure & $\begin{array}{c}\text { Spherical alloy } \\
1.0: 0.85\end{array}$ & $\begin{array}{c}\text { Conventional alloy } \\
1.0: 1.0\end{array}$ \\
\hline $\begin{array}{l}10 \mathrm{~kg} / \mathrm{cm}^{2} \\
60\end{array}$ & $\begin{array}{l}0.54 \mu \mathrm{A} \\
0.52\end{array}$ & $\begin{array}{l}0.79 \mu \mathrm{A} \\
0.68\end{array}$ \\
\hline
\end{tabular}

TABLE 15

Corrosion Current of Conventional Alloy

A-M ratio: $1.0: 1.0$

Child saliva

\begin{tabular}{|c|c|c|c|c|}
\hline \multirow{2}{*}{$\begin{array}{l}\text { Condensation } \\
\text { pressure }\end{array}$} & \multicolumn{3}{|c|}{ Voltage } & \multirow{2}{*}{ Residual mercury } \\
\hline & $0.1 \mathrm{v}$ & $0.2 \mathrm{v}$ & $0.3 \mathrm{v}$ & \\
\hline $\begin{array}{l}10 \mathrm{~kg} / \mathrm{cm}^{2} \\
60\end{array}$ & $\begin{array}{l}0.37 \mu \mathrm{A} \\
0.31\end{array}$ & $\begin{array}{l}0.60 \mu \mathrm{A} \\
0.53\end{array}$ & $\begin{array}{l}0.86 \mu \mathrm{A} \\
0.75\end{array}$ & $\begin{array}{l}50.0 \% \\
46.0\end{array}$ \\
\hline
\end{tabular}




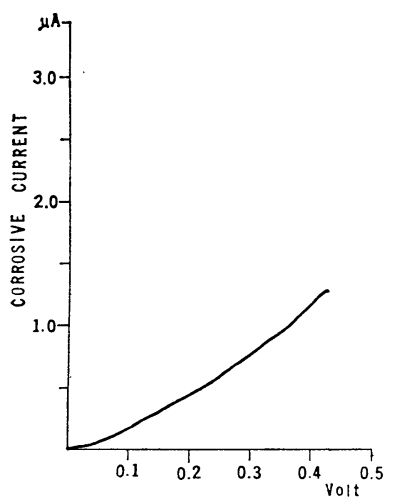

Fig. 45. Conventional $1.0: 1.0 \quad 10 \mathrm{~kg} / \mathrm{cm}^{2}$ Child saliva

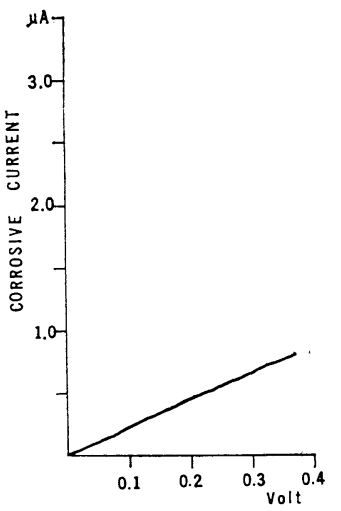

Fig. 47. Spherical $1.0: 0.8510 \mathrm{~kg} / \mathrm{cm}^{2}$ Child saliva

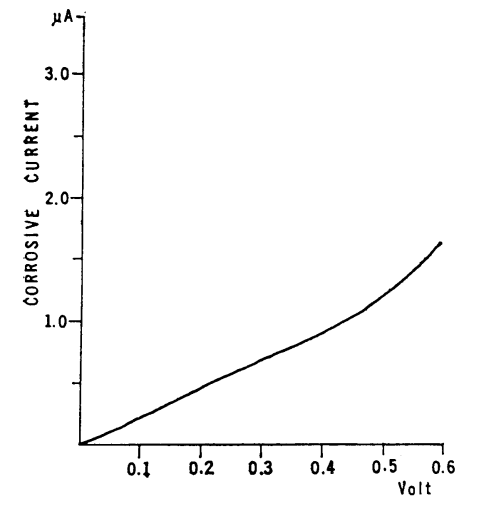

Fig. 46. Conventional $1.0: 1.0 \quad 60 \mathrm{~kg} / \mathrm{cm}^{2}$ Child saliva

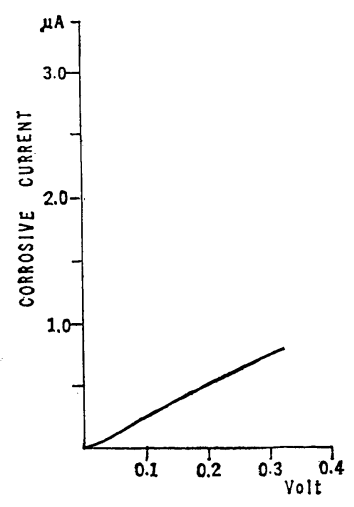

Fig. 48. Spherical $1.0: 0.8560 \mathrm{~kg} / \mathrm{cm}^{2}$ Child saliva

TABLE 16

Corrosion Current of Spherical Alloy

A-M ratio: $1.0: 0.85$

Child saliva

\begin{tabular}{|c|c|c|c|c|}
\hline \multirow{2}{*}{$\begin{array}{l}\text { Condensation } \\
\text { pressure }\end{array}$} & \multicolumn{3}{|c|}{ Voltage } & \multirow{2}{*}{ Residual mercury } \\
\hline & $0.1 \mathrm{v}$ & $0.2 \mathrm{v}$ & $0.3 \mathrm{v}$ & \\
\hline $\begin{array}{l}10 \mathrm{~kg} / \mathrm{cm}^{2} \\
60\end{array}$ & $\begin{array}{l}0.31 \mu \mathrm{A} \\
0.25\end{array}$ & $\begin{array}{l}0.49 u \mathrm{~A} \\
0.50\end{array}$ & $\begin{array}{l}0.70 \mu \mathrm{A} \\
0.71\end{array}$ & $\begin{array}{l}45.8 \% \\
43.5\end{array}$ \\
\hline
\end{tabular}

\subsubsection{Effect on Spherical Amalgam.}

The same alloy-mercury ratio of 1.0:0.85 was adopted as in the case of adult saliva and the measurements were made under two condensation pressures of $10 \mathrm{Kg} / \mathrm{cm}^{2}$ and $60 \mathrm{Kg} / \mathrm{cm}^{2}$ (Figs. 47, 48). Table 16 compares the two mean current readings. As is 
the case with the artificial saliva, changes in condensation pressure have no appreciable effect on the corrosive tendency of spherical amalgam.

\subsubsection{Comparison of Spherical and Conventional Amalgams.}

With the child saliva as a corrosive agent, Table 17 gives a comparison of the corrosive tendencies on the part of conventional and spherical amalgams at $0.2 \mathrm{~V}$. as is shown in the table, while the corrosive tendency of spherical remains more or less unchanged within a range of 10 to $60 \mathrm{Kg} / \mathrm{cm}^{2}$ condensation pressure, that of conventional amalgam is greatly influenced by whether a condensation pressure is large or small.

TABLE 17

Comparison of Corrosion Current of Spherical and Conventional Alloy at 0.2 Volt

Child saliva

\begin{tabular}{c|c|c}
\hline \hline Condensation pressure & $\begin{array}{c}\text { Spherical alloy } \\
1.0: 0.85\end{array}$ & $\begin{array}{c}\text { Conventional alloy } \\
1.0: 1.0\end{array}$ \\
\cline { 1 - 2 } $10 \mathrm{~kg} / \mathrm{cm}^{2}$ & $0.49 \mu \mathrm{A}$ & $0.60 \mu \mathrm{A}$ \\
60 & 0.50 & 0.53
\end{tabular}

The relationship of the corrosion resistancy and the condensation pressure on both material showed similar results as is seen in adult saliva.

The obvious superiority of the corrosion tendency of the spherical amalgam was shown in lower pressure as $10 \mathrm{Kg} / \mathrm{cm}^{2}$ in comparison with conventional amalgam with same pressure.

\section{Discussion}

NAGAI had earlier entertained an idea that an electrochemical method would be most effective in the determination of the corrosive tendency of dental alloys and introduced the use of polarographic method for this purpose. TSURUMAKI[10] and $\mathrm{HABU}[11]$ published interesting results respectively under the guidance of NAGAI, in which they carried out detailed studies on the corrosive tendency of various alloys used in dentistry. To put it in a few words, this method consists in measuring the volume of electric currents generating at a low bath voltage below the decomposition voltage and in determining the corrosive tendency of a given dental alloy by obtaining the bath voltage-current curves.

This method was employed in the present study and here the following items are to be discussed in consideration of the fact that there exist differences in various salivae as electrolytic substances and structural differences of test specimens as well.

\subsection{Differences owing to Different Kinds of Saliva.}

In regarding the saliva as an electrolytic substance, its $\mathrm{pH}$ levels should be, first of all, taken into consideration. The human saliva is generally held to be within a $\mathrm{pH}$ range of 5.0 to 8.0 [13], while the infant saliva is said to be inclined somewhat toward the alkali side [14[. Although the $\mathrm{pH}$ level of an individual saliva is more or less constant, there is observed a wide disparity from individual to individual in this respect. This phenomenon is also true in the passage of time.

As has been mentioned in the section concerning the experimental method, the 
infant and adult salivae respectively registered the $\mathrm{pH}$ readings of $6.4-7.6$ and $6.2-7.8$ and though they were a little inclined toward the alkali side, there was not much difference between the two.

In our preparatory procedures, an effort was made to lock in into the corrosive action of the adult saliva on account of its higher $\mathrm{pH}$ level but there was not observed any appreciable corrosive effect. As compared with the adult saliva, the infant saliva gave a still lower corrosive action and though we attribute this to the difference of ingredients and composition of the infant saliva, a definite study on this subject is

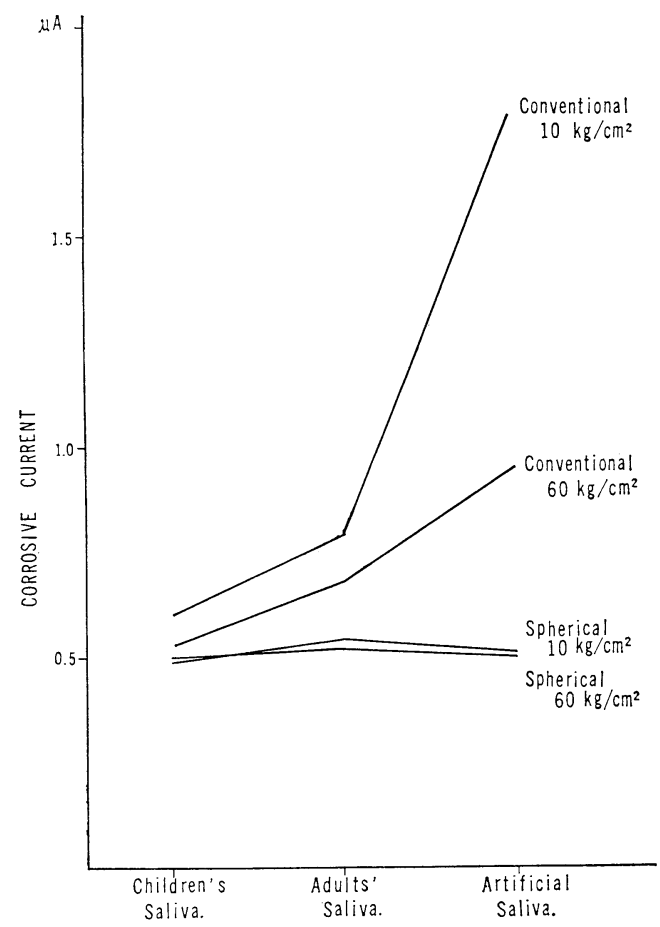

Fig. 49. Comparison of corrosion current at 0.2 Volt in artificial, adult, and child saliva

TABLE 18

Comparison of Corrosion Current of Spherical and Conventional Alloys at 0.2 Volt in Three Different Corrosive Agents

\begin{tabular}{|c|c|c|c|c|c|c|}
\hline \multirow{3}{*}{$\begin{array}{l}\text { Condensation } \\
\text { pressure }\end{array}$} & \multicolumn{2}{|c|}{ Artificial saliva } & \multicolumn{4}{|c|}{ Human salivae. } \\
\hline & \multirow{2}{*}{ Sphe. } & \multirow{2}{*}{ Conv. } & \multicolumn{2}{|c|}{ Child } & \multicolumn{2}{|c|}{ Adult } \\
\hline & & & Sphe. & Conv. & Sphe. & Conv. \\
\hline $10 \mathrm{~kg} / \mathrm{cm}^{2}$ & $0.51 \mu \mathrm{A}$ & $1.79 \mu \mathrm{A}$ & $0.49 \mu \mathrm{A}$ & $0.60 \mu \mathrm{A}$ & $0.54 \mu \mathrm{A}$ & $0.79 u \mathrm{~A}$ \\
\hline 60 & 0.50 & 0.95 & 0.50 & 0.53 & 0.52 & 0.68 \\
\hline
\end{tabular}

A-M ratio: Spherical 1.0:0.85

Conventional $1.0: 1.0$ 
regrettably lacking. At any rate, different kinds of the saliva bring about pronounced differences in the corrosion of conventional amalgam but no appreciable change in the corrosion is noted with spherical amalgam, as is seen in Fig. 49 and Table 18. Therefore we are inclined to the view that difference in the corrosive tencency should be sought in the difference of these amalgams rather in the difference of salivae in question.

\subsection{Differences in Corrosive Tendencies of Conventional and Spherical Amalgams.}

The mercury content of dental amalgam was pointed out by HABU [11] to be an important factor in influencing the electric currents. In our test specimens, an alloymercury ratio was 1.0:1.0 in the case of conventional amalgam with the volumes of residual mercury being $50.0 \%\left(10 \mathrm{Kg} / \mathrm{cm}_{2}\right)$ and $46.0 \%\left(60 \mathrm{Kg} / \mathrm{cm}_{2}\right)$, while a ratio of $1.0: 0.85$ was used with spherical amalgam in which the volumes of residual mercury were $45.8 \%\left(10 \mathrm{Kg} / \mathrm{cm}_{2}\right)$ and $43.5 \%\left(60 \mathrm{Kg} / \mathrm{cm}_{2}\right)$ respectively. Although the general tendency was that the volume of residual mercury decreased in proportion to an increase in condensation pressure, posssible influence by the volume of residual mercury was certainly negligible in the case of the human saliva. As compared with the human saliva, on the other hand, effect of residual mercury of conventional amalgam was apparent and large differences were noted with electric currents in the case of artificial saliva.

The authors were led to seek the cause of these phenomena in the structural differences of crystals on the part of these alloys, i. e., the volume of local electric currents influenced by the crystallogical differences on the corrosive surfaces. The crystallogical structure of dental amalgam is thought that there exist two phases of $\gamma_{1}$ and $\gamma_{2}$ which are made up of $\mathrm{Ag}_{2} \mathrm{Hg}_{3}$, and $\mathrm{Sn}_{7} \mathrm{Hg}$, and additional $\beta_{2}$ with the alloy particles as their nuclei and mercury is found among them in the residual state. Since spherical amalgam has the structure of spherical particles as is implied by its name while conventional amalgam is made up of irregular particles, it is natural that there should be definite difference between the two structures. This difference has bearings on the volume of local electric currents and this also explains that pronounced corrosive tendency took place in conventional amalgam because of its larger particle surfaces.

\subsection{Effect of Different Condensation Pressures on Amalgam Corrosion.}

A deteiled report was already published by NAGAI et al. [5] concerning the effect of condensation pressures on spherical amalgam. The report definitely established that spherical amalgam can be very compactly packed even under a low condensation pressure, whereas with conventional amalgam a condensation pressure larger than $60 \mathrm{Kg} / \mathrm{cm}^{2}$ is required to effect a similar compact packing. As shown in Figs. 50 and 51 and Table 18, these results are also confirmed by our present study: while $1.79 \mu \mathrm{A}$ (10 $\left.\mathrm{Kg} / \mathrm{cm}^{2}\right)$ decreased appreciably to $0.95 \mu \mathrm{A}\left(60 \mathrm{Kg} / \mathrm{cm}^{2}\right)$ in the case of conventional amalgam triturated at $1.0: 1.0$, spherical amalgam triturated at $1.0: 0.85$ merely registered a decrease of $0.56 \mu \mathrm{A}\left(5 \mathrm{Kg} / \mathrm{cm}^{2}\right)$ to $0.50 \mu \mathrm{A}\left(60 \mathrm{Kg} / \mathrm{cm}^{2}\right)$, both tested by means of artificial saliva. When $5 \mathrm{Kg} / \mathrm{cm}^{2}$ pressure for spherical is contrasted with $60 \mathrm{Kg} / \mathrm{cm}^{2}$ pressure of conventional amalgam, we note that the former has a far better corrosion-resistancy, thus a difference between the two condensation pressures being quite large.

On the strength of these considerations, it may be thought that effect of the condensation pressure is not to be sought in the residual mercury alone, but also in the crystallogical structure of a given alloy surface. This is demonstrated by the fact that spherical amalgam (triturated under a pressure of $10 \mathrm{Kg} / \mathrm{cm}^{2}$ in $1.0: 0.85$ ) gave $0.49 \mu \mathrm{A}$, 


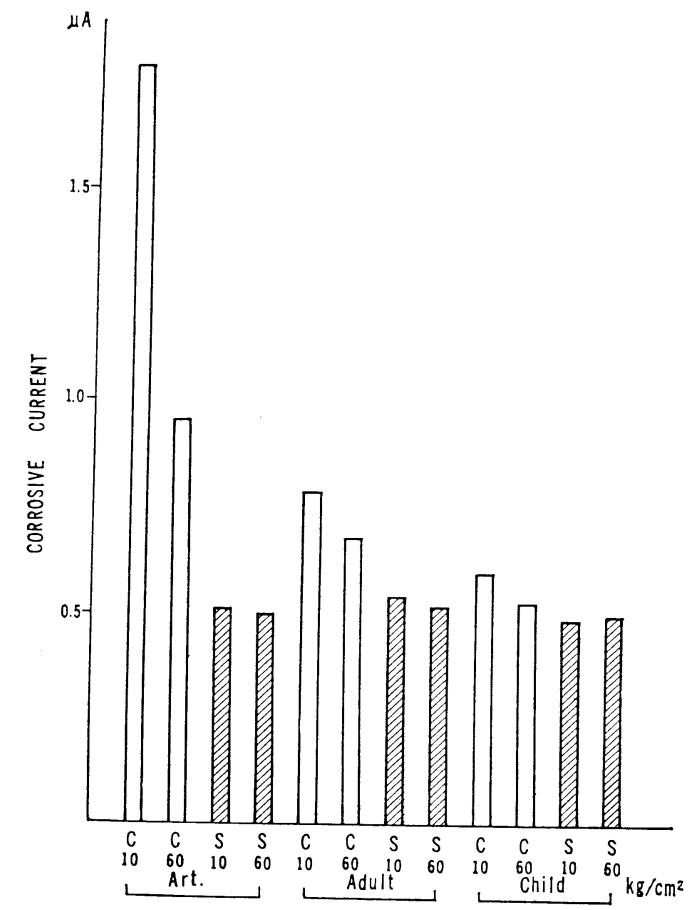

Fig. 50. Comparison of corrosion current at 0.2 Volt

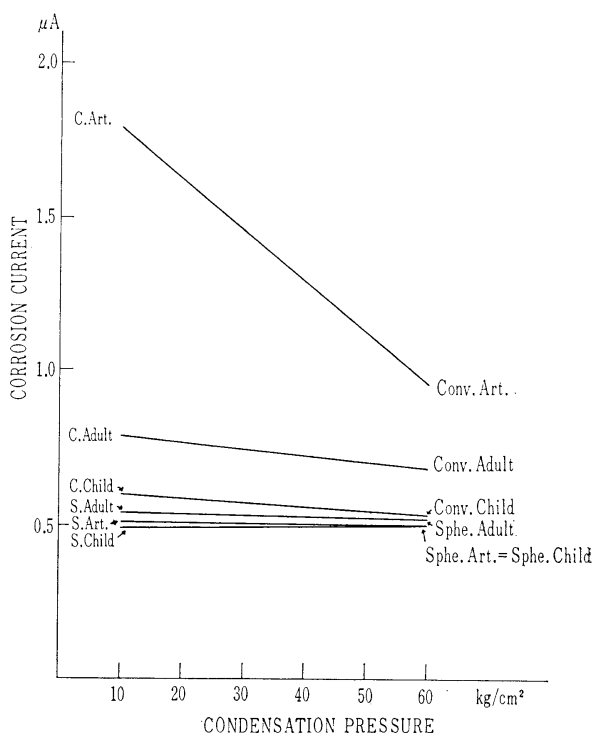

Fig. 51. Comparison of corrosive current according to the condensation pressure from 10 to $60 \mathrm{~kg} / \mathrm{cm}^{2}$ in artificial, adult, child saliva

while conventional amalgam (triturated under a pressure of $60 \mathrm{Kg} / \mathrm{cm}^{2}$ in $1.0: 1.0$ ) gave $0.52 \mu \mathrm{A}$. As is shown by these comparative figures in Table 18 and Fig. 49, we note a definite differece in the corrosion of the two different kinds of amalgam alloys but nevertheless we cannot ascribe this difference to a condensation pressure alone on the ground that different salivae are attended by different corrosive behaviors.

In other words, this kind of complex corrosive pattern should be accounted for by not only change in the volume of residual mercury but also by the uniformity and compactness of amalgam restoration as well as the electrolytic property of a corrosive agent involved.

\section{Conclusions}

As a result of their comparative study on the corrosive tendencies of conventional and spherical amalgams, the authors came to arrive at the conclusions as follows.

1) In terms of the infant, adult and artificial salivae, the corrosive resistancy of spherical amalgam was definitely advantageous over that of conventional amalgam.

2) As compared with the fact that the corrosion of spherical amalgam showed no appreciable difference within a range of 10 to $60 \mathrm{Kg} / \mathrm{cm}^{2}$ condensation pressures, that of conventional amalgam was heavily influenced by a condesation pressure involved.

3) Much clinical implication was established by the fact that spherical amalgam packed under a low condensation pressure gave a better corrosion resistancy than conventional amalgam packed under a high condensation pressure. This conclusion, we 
believe, will prove quite beneficial to the daily routine of dental practitioners.

4) As for spherical amalgam, there was not measured any appreciable difference within a range of $1.0: 0.70$ to $1.0: 0.85$ alloy-mercury ratios with regard to its corrosive patterns.

5) While conventional amalgam was shown to have different corrosive behaviors depending upon the kind of salivae involved, spherical amalgam gave no appreciable differences on this account.

6) On the strength of the foregoing findings, spherical amalgam possesses high clinical advantages as a dental restorative material and these advantages will be particularly noteworthy in the field of pedodontics in which any undue strong pressure should be avoided as much as possible.

\section{References}

[1] Probst, R. L., Karp, P. I., Sayre, C. H. and Beebe, E. R. Jr. : Assingors to Federal Mogul Bower Bearings, Inc., Detroit, Michigan, Atomizing nozzle and fouring cap assembly for the manufacture of metal powders, US patent No- 2, 968, 062 (January 17, 1961)

[2] Demaree, N. C. and TAYLOR, D. F.: Properties of dental amalgam made from spherical alloy particles, J. dent. Res., 41 : 890-906, 1962.

[3] KorAn, A. and Asgar, K. : Usage of spherical amalgam alloys in dentistry, presented at 1965 I. A. D. R. annual meeting.

[4] Eden, G. T. and Waterstrat, K. M. : Effect of packing pressure on tensile strength of commercial and spherical amalgams, presented at 1965 I. A. D. R. annual meeting.

[5] Nagai, K., Ohashi, M. and MiYazU, H. : Studies on spherical amalgam alloy in the light of dental technology. J. Nihon Univ. Sch. Dent. 8 : 149, 1966.

[6] Nagai, K. and OHASHI, M. : Studies on the dimensional change of dental amalgam. J. Nihon Univ. Sch. Dent., 9: 1, 1966.

[7] NagAi, K. and OHASHI, M. : Three studies on a newly developmed spherical amalgam in Japan and suggestions for its specification. J. Nihon Univ. Sch. Dent. 9: 2, 43. 1967.

[ 8 ] NagAi, K., Ohashi, M. and Hasegawa, K. : Comparative study on the marginal strength of conventional and spherical amalgam alloys. Invention of a device for the Measurement of marginal strength. J. Nihon Univ. Sch. Dent. 9 : 2, 49, 1967.

[9] Nagai, K. and Yamamoto, Y.: On the effect of metal corrosion by R. G. Y. test and corrosive action of liquids, Shikai-tembo, 12: 437-441, 1955 (in Japanese).

[10] TsRumaki, K. : Determination of the corrosive tendency of dental alloys by the polarography, Shika-Geppo, 34: 2, 74-119, 1960.

[11] HABU, H. : A polarographic study on the corrosion tendency of dental amalgam. J. Nihon Univ. Sch. Dent. 6: 1, 6-16, 1964.

[12] Greenwood, J. N., Dowan, C. H. and Wonder, H. K. : An investnigation of failure of stainless steel inlay retention post. Austral, J. Dent., 41:3, 73-84, 1937.

[13] Oshikane, A. et al. : Dental biochemistry, Medico-dento-pharmacological Publ. Co., Tokyo (1966).

[14] AFONSKY, D. : Saliva and its relation to oral hygiene. Univ. Alabama press, Birmingham, Alabma, p. 235, (1961). 